\title{
Demonstration of Antiallodynic Effects of the Cyclooxygenase-2 Inhibitor Meloxicam on Established Diabetic Neuropathic Pain in Mice
}

\author{
Satoko Kimura ${ }^{1}$ and Hitoshi Kontani ${ }^{1, *}$ \\ ${ }^{1}$ Department of Pharmacology, Faculty of Pharmaceutical Sciences, Hokuriku University, \\ Kanagawa-machi, Kanazawa 920-1181, Japan
}

Received January 12, 2009; Accepted April 7, 2009

\begin{abstract}
Only a few experimental studies have demonstrated the effectiveness of some cyclooxygenase-2 (COX-2) inhibitors for neuropathic pain in diabetic animals. In this study we investigated the usefulness of one such COX-2 inhibitor, meloxicam, for treatment of established diabetic neuropathic pain in mice. Intraperitoneal and perineural injection, but not intrathecal injection, of meloxicam elevated the lowered threshold in the von Frey test. These results suggest that meloxicam exerts antiallodynic effects on established neuropathic pain in diabetic mice, and that the site of its action is peripheral. Meloxicam may therefore be a potentially useful drug for treatment of diabetic neuropathic pain.
\end{abstract}

Keywords: diabetic neuropathic pain, cyclooxygenase-2 (COX-2) inhibitor, antiallodynic effect

Diabetes is frequently associated with painful polyneuropathies. However, conventional analgesics that relieve acute pain cannot exert sufficient effects on neuropathic pain associated with diabetes, and no potentially effective drug for treatment of this type of pain is currently available. Although the etiology of this painful diabetic polyneuropathy is still not completely understood, it is generally agreed that both peripheral and central sensitization are involved in the pathogenesis of neuropathic pain, and prostaglandins sensitize the nociceptive system at peripheral as well as central sites, chiefly the spinal cord (1).

In diabetic rats, an increase of cyclooxygenase-2 $(\mathrm{COX}-2)$ protein in the lumbar spinal cord and sciatic nerve has been demonstrated $(2,3)$. However, few reports have suggested the effectiveness of selective COX-2 inhibitors against the development and maintenance of hyperalgesia in diabetic rats $(4,5)$. Meloxicam is a clinically employed nonsteroidal anti-inflammatory drug with a COX-2-preferential action (6). In rats with neuropathic pain due to peripheral nerve injury, meloxicam attenuates the development and maintenance of allodynia and hyperalgesia (7). However, to our knowledge, no study of meloxicam's action in diabetic

*Corresponding author. h-kontani@hokuriku-u.ac.jp Published online in J-STAGE on June 5, 2009 (in advance) doi: $10.1254 /$ jphs.09006SC neuropathic pain has been reported. Therefore, we investigated the effects of meloxicam on established neuropathic pain in diabetic mice.

All experimental protocols used were approved by the Animal Care and Use Committee of Hokuriku University, and procedures were conducted in accordance with the guidelines of the National Institutes of Health and The Japanese Pharmacological Society.

Male ddY mice, 7-9 week of age, were used in all experiments. For assessment of mechanical allodynia, mice were placed singly in an individual $200-\mathrm{ml}$ beaker with a wire-mesh bottom. Eight von Frey filaments (North Coast Medical, Morgan Hill, CA, USA) with approximately equal logarithmic incremental bending forces $(0.02-1.4 \mathrm{~g})$ were applied to the plantar surface of the hind paw. The paw withdrawal threshold was determined by the up-down method of Dixon (8) and Chaplan et al. (9).

Diabetes was induced by a single intraperitoneal (i.p.) injection of streptozotocin (STZ, $200 \mathrm{mg} / \mathrm{kg}$ ) dissolved in saline. The blood glucose concentration was measured using a glucometer 7 days after STZ injection from blood samples obtained by tail prick. The mice were defined as diabetic when their blood glucose concentration exceeded $350 \mathrm{mg} / \mathrm{dl}$. Mechanical threshold was assessed using von Frey filaments just before and 14 days after STZ injection. Partial sciatic nerve ligation was done according to the original method 
of Seltzer et al. (10). Under pentobarbital $(60 \mathrm{mg} / \mathrm{kg}$, i.p.) anesthesia, the left sciatic nerve was exposed at the upper-thigh level, and the dorsal third to half of the sciatic nerve was tightly ligated. The wound was then sutured and the mice were allowed to recover in their home cages. Mechanical threshold was assessed just before and 7 days after sciatic nerve ligation. Fourteen days after STZ injection or 7 days after sciatic nerve ligation, mice with a threshold of less than $0.1 \mathrm{~g}$ in the von Frey test were considered to be developing tactile allodynia and used in the following experiments.

STZ, meloxicam sodium, and ibuprofen sodium were from Sigma (St. Louis, MO, USA). Pentobarbital sodium (Nembutal ${ }^{\mathbb{R}}$ injection) was from Dainippon Sumitomo Pharma (Osaka). STZ, meloxicam, and ibuprofen were dissolved in saline. Pentobarbital was diluted with saline. When given i.p., the drugs were administered in a volume of $0.1 \mathrm{ml} / 10 \mathrm{~g}$ body weight.
For intrathecal (i.t.) injection, the drugs were administered in a volume of $5 \mu \mathrm{l}$ via a 27 -gauge needle, which was inserted into the subarachnoid space through the intervertebral foramen between L5 and L6, according to the method described by Hylden and Wilcox (11). For perineural (p.n.) injection, we modified the method for rats described by Thalhammer et al. (12) in order to apply it to mice. Each mouse was restrained in a cotton glove, and then a 27-gauge needle was inserted near the greater trochanter and advanced to the ossa coxae so that the tip of the needle was located near the site at which the sciatic nerve had been ligated in Seltzer-model mice. The tip of the needle was also located at the same site in diabetic mice. The injection volume was $5 \mu$ l. When sciatic nerve ligation was performed in the Seltzermodel mice for p.n. injection, the perineurium was cut along the nerve fibers for about $5 \mathrm{~mm}$ with a $30 \mathrm{G}$ needle to expose the nerve fully to meloxicam, and
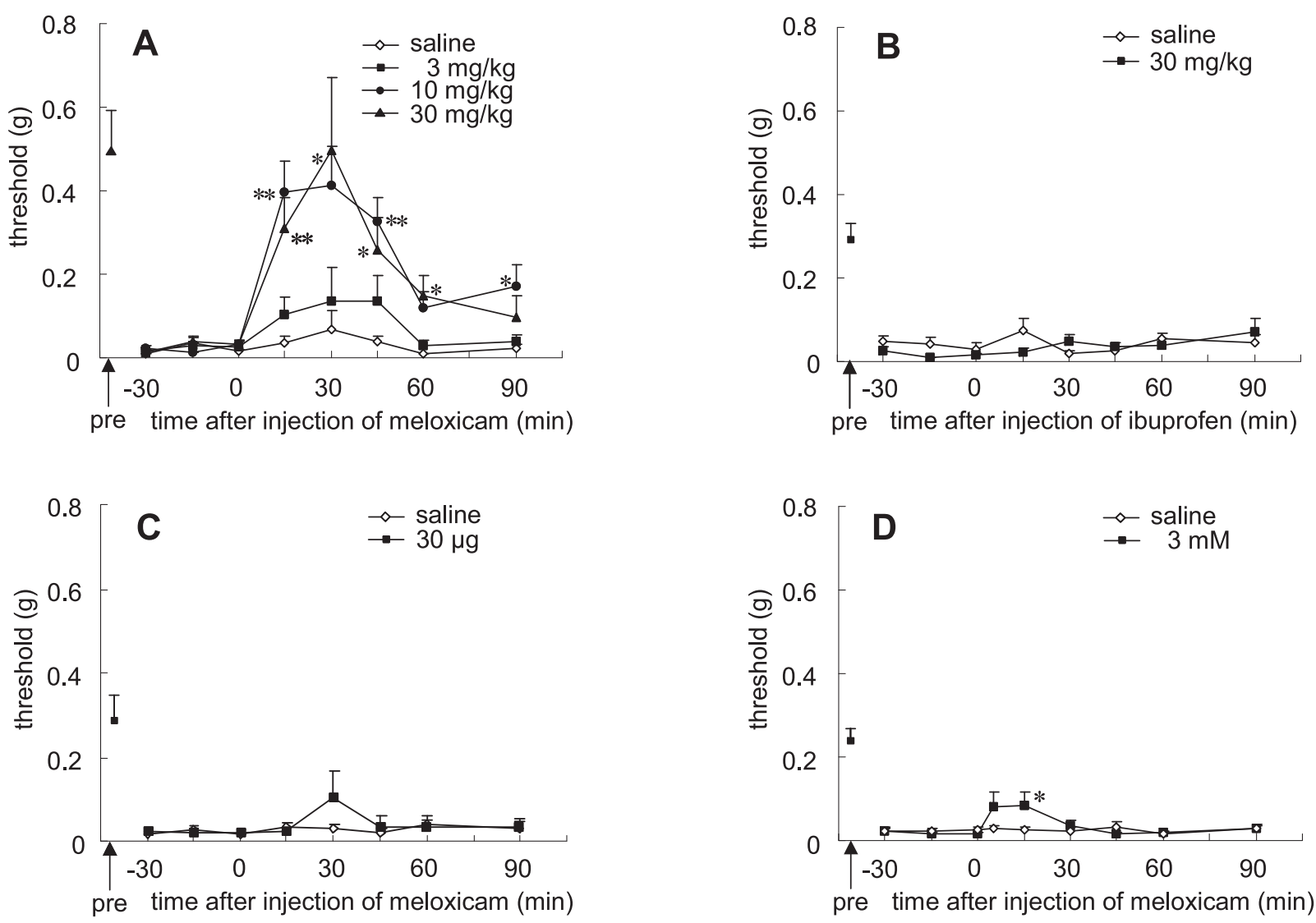

Fig. 1. Effects of meloxicam and ibuprofen on allodynia in diabetic mice. Meloxicam was injected i.p., i.t., or p.n.; and the effects are shown in A, C, and D, respectively. Ibuprofen was injected i.p., and its effects are shown in B. Drugs were injected at time 0 . Each value represents the mean \pm S.E.M. of 6 to 8 mice per group. Ordinates: mean thresholds $(\mathrm{g})$ as assessed by the von Frey test. Abscissa: time in minutes after drug injection 14 days after STZ $(200 \mathrm{mg} / \mathrm{kg}$, i.p.) injection. Before STZ injection, the threshold was measured and the symbol showing this level is indicated by an arrow and the term "pre" on the abscissa. The paired or unpaired $t$-test was used to compare the data between two groups, and the $t$-test with the Bonferroni correction following one-way analysis of variance was used for multiple comparisons of the control and treated groups. ${ }^{*} P<0.05$ and $* * P<0.01$ vs. control at the respective time. 
the perineurium cutting procedure was done under pentobarbital anesthesia 7 days after STZ injection in the diabetic mice receiving p.n. injection.

All data are expressed as the mean \pm S.E.M. The paired or unpaired $t$-test was performed as appropriate, and the $t$-test with Bonferroni correction following one-way analysis of variance (ANOVA) was used for multiple comparisons. Differences at $P<0.05$ were considered to be significant.

Meloxicam (10 and $30 \mathrm{mg} / \mathrm{kg}$, i.p.) significantly raised the threshold values of the withdrawal response in diabetic mice (Fig. 1A, one-way ANOVA, $n=6$ ). There was no significant difference between the two values for $90 \mathrm{~min}$ after injection of meloxicam (10 and 30 $\mathrm{mg} / \mathrm{kg}$ ). The threshold before injection of STZ was not significantly different from the value $15 \mathrm{~min}$ after injection of meloxicam $(30 \mathrm{mg} / \mathrm{kg})$ in the diabetic mice (paired $t$-test). On the other hand, ibuprofen $(30 \mathrm{mg} / \mathrm{kg}$, i.p.) did not increase the threshold value for $90 \mathrm{~min}$ in diabetic mice (Fig. 1B). I.t. injection of meloxicam (30 $\mu \mathrm{g}$ /animal) did not change the threshold value (Fig. 1C). Although p.n. injection of meloxicam (3 mM) raised the value, the maximum value was very small as compared with that after i.p. injection (Fig. 1D, unpaired $t$-test, $\mathrm{n}=6-8$ ).

I.p. injection of meloxicam (10 or $30 \mathrm{mg} / \mathrm{kg}$ ) significantly raised the threshold values of the withdrawal response in Seltzer-model mice (one-way ANOVA, $n=6$ ), and there was no significant difference between the two threshold values for $90 \mathrm{~min}$ after injection of meloxicam (10 and $30 \mathrm{mg} / \mathrm{kg}$, i.p.) (Fig. 2A). The threshold value before sciatic nerve injury was not significantly different from that at $15 \mathrm{~min}$ after injection of meloxicam $(30 \mathrm{mg} / \mathrm{kg})$ in the Seltzer-model mice (paired $t$-test). Also in this model, ibuprofen $(30 \mathrm{mg} / \mathrm{kg}$, i.p.) did not exert an antiallodynic effect (Fig. 2B). I.t.
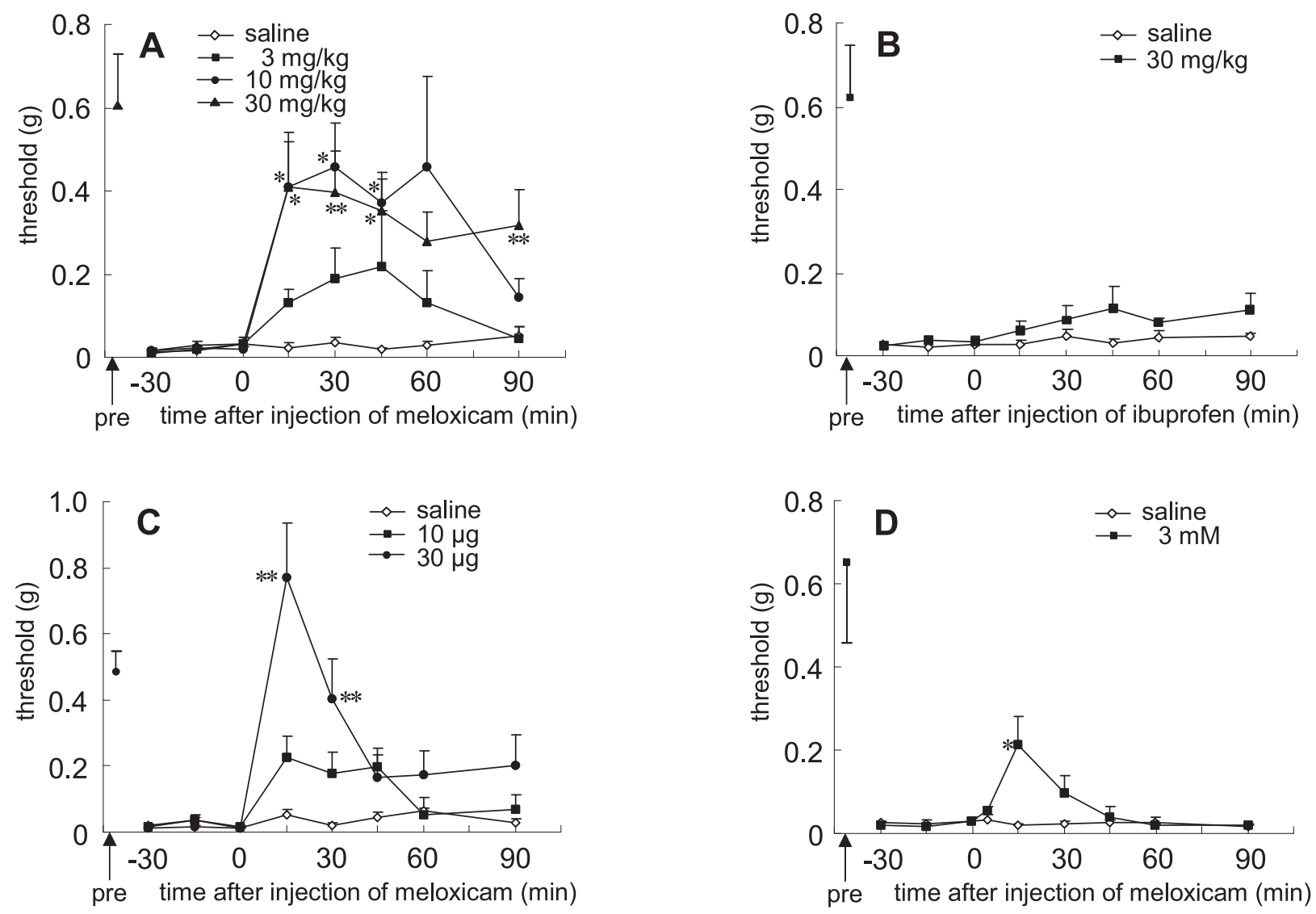

Fig. 2. Effects of meloxicam and ibuprofen on allodynia in Seltzer-model mice. Meloxicam was injected i.p., i.t., or p.n.; and the effects are shown in A, C, and D, respectively. Ibuprofen was injected i.p., and its effects are shown in B. Drugs were injected at time 0 . Each value represents the mean \pm S.E.M. of 6 to 8 mice per group. Ordinates: mean thresholds (g) as assessed by the von Frey test. Abscissa: time in minutes after drug injection 7 days after sciatic nerve injury. Before the surgical procedure, the threshold was measured and the symbol showing this level is indicated by an arrow and the term "pre" on the abscissa. The paired or unpaired $t$-test was used to compare the data between two groups, and the $t$-test with the Bonferroni correction following one-way analysis of variance was used for multiple comparisons of the control and treated groups. ${ }^{*} P<0.05$ and ${ }^{* *} P<0.01$ vs. control at the respective time. 
and p.n. injection of meloxicam $(30 \mu \mathrm{g}$ and $3 \mathrm{mM}$, respectively) induced antiallodynic effects (Fig. 2C, one-way ANOVA, $\mathrm{n}=6-7$; Fig. $2 \mathrm{D}$, unpaired $t$-test, $\mathrm{n}=6$ ).

In the present study, we demonstrated that i.p.injected meloxicam exerted an antiallodynic effect in diabetic mice. The fact that the threshold elevation by meloxicam did not exceed the pretreatment value (Figs. 1A and 2A) suggests that the drug may inhibit the exaggerated COX-2 activity in both mouse models (2, 3). Although i.t.-injected meloxicam (30 $\mu \mathrm{g} / \mathrm{animal})$ hardly exerted an antiallodynic effect in diabetic mice, it exerted a clear effect in Seltzer-model mice (Figs. 1C and $2 \mathrm{C}$ ). From these results, it seems that meloxicam acts mainly at peripheral sites to exert its antiallodynic effects in diabetic mice, whereas it acts at both peripheral sites and the spinal cord in Seltzer-model mice. As other COX-2 inhibitors with $\mathrm{IC}_{50}$ values for COX-2 about five times lower than that of meloxicam have been reported to attenuate hyperalgesia in diabetic rats $(4,13)$, a higher dose of meloxicam may exert antiallodynic activity in diabetic mice. Moreover, for evaluation of diabetic neuropathic pain, those authors used an electronic von Frey anesthesimeter, whereas we employed a series of von Frey filaments that were applied manually. The difference between their results and ours may have been attributable to this difference in methodology. We investigated the antiallodynic effects of meloxicam in diabetic mice at a dose that was able to relieve the allodynia induced by the Seltzermodel. As the level of meloxicam in the central nervous system after peroral administration is very low (14), systemically administered meloxicam exerted its antiallodynic effect at peripheral sites in both mouse models, and the peripheral potency of meloxicam was almost the same in the two models. P.n. administration of meloxicam $(3 \mathrm{mM})$ partially increased the threshold of the withdrawal response in both mouse models (Figs. 1D and 2D). Diabetic polyneuropathy induces pathological changes in the whole peripheral nervous system. Also in Seltzer-model mice, the pathological changes were not restricted to the vicinity of the injured site. P.n.-injected meloxicam reached the nerve fibers via a tear in the perineurium, and therefore its distribution was restricted to the area around the injection site. The antiallodynic effect of meloxicam is not due to its local anesthetic activity, since at the same concentration meloxicam did not reduce the amplitude of the compound action potentials in isolated sciatic nerves of mice (our preliminary observation). These results suggest that meloxicam may exert its antiallodynic effect through inhibition of exaggerated COX-2 activity in peripheral nerves in both mouse models. Peripheral nerve injury or diabetic peripheral neuropathy leads to neuropathic pain, which is induced by nociceptor sensitization of primary afferent neurons.

Ibuprofen $(30 \mathrm{mg} / \mathrm{kg}$, i.p.) did not show an antiallodynic effect in both mouse models, even though this dose is effective against inflammation-induced hyperalgesia (15). As the $\mathrm{IC}_{80}$ of COX-2 for ibuprofen is approximately $10-75$ times higher than that for meloxicam (16), the dose we used in the present experiment would have been insufficient to inhibit the COX-2 activity. These results also suggest that elevation of the COX-2 level in both mouse models is related to induction of allodynia.

As the values of von Frey filaments used in this study were not continuous, we also analyzed all the data using non-parametric methods. However, the statistical evaluations obtained by the parametric method were consistent with those obtained non-parametrically.

In conclusion, we have demonstrated the effectiveness of meloxicam for amelioration of established allodynia in mice with diabetes. These results indicate that meloxicam shows promise as a drug for treatment of diabetic neuropathic pain.

\section{References}

1 Schaible HG, Ebersberger A, Von Banchet GS. Mechanisms of pain in arthritis. Ann N Y Acad Sci. 2002;966:343-354.

2 Freshwater JD, Svensson CI, Malmberg AB, Calcutt NA. Elevated spinal cyclooxygenase and prostaglandin release during hyperalgesia in diabetic rats. Diabetes. 2002;51:2249-2255.

3 Kellogg AP, Wiggin TD, Larkin DD, Hayes JM, Stevens MJ, Pop-Busui R. Protective effects of cyclooxygenase-2 gene inactivation against peripheral nerve dysfunction and intraepidermal nerve fiber loss in experimental diabetes. Diabetes. 2007;56:2997-3005.

4 Matsunaga A, Kawamoto M, Shiraishi S, Yasuda T, Kajiyama $\mathrm{S}$, Kurita $\mathrm{S}$, et al. Intrathecally administered COX-2 but not COX-1 or COX-3 inhibitors attenuate streptozotocin-induced mechanical hyperalgesia in rats. Eur J Pharmacol. 2007;554:1217.

5 Bujalska M, Tatarkiewicz J, de Cordé A, Gumulka SW. Effect of cyclooxygenase and nitric oxide synthase inhibitors on streptozotocin-induced hyperalgesia in rats. Pharmacology. 2008;81:151-157.

6 Noble S, Balfour JA. Meloxicam. Drugs. 1996;51:424-430; discussion 431-432.

7 Takeda K, Sawamura S, Tamai H, Sekiyama H, Hanaoka K. Role for cyclooxygenase 2 in the development and maintenance of neuropathic pain and spinal glial activation. Anesthesiology. 2005;103:837-844.

8 Dixon WJ. Efficient analysis of experimental observations. Annu Rev Pharmacol Toxicol. 1980;20:441-462.

9 Chaplan SR, Bach FW, Pogrel JW, Chung JM, Yaksh TL. Quantitative assessment of tactile allodynia in the rat paw. J Neurosci Methods. 1994;53:55-63.

10 Seltzer Z, Dubner R, Shir Y. A novel behavioral model of 
neuropathic pain disorders produced in rats by partial sciatic nerve injury. Pain. 1990;43:205-218.

11 Hylden JL, Wilcox GL. Intrathecal morphine in mice: a new technique. Eur J Pharmacol. 1980;67:313-316.

12 Thalhammer JG, Vladimirova M, Bershadsky B, Strichartz GR. Neurologic evaluation of the rat during sciatic nerve block with lidocaine. Anesthesiology. 1995;82:1013-1025.

13 Marnett LJ, Kalgutkar AS. Design of selective inhibitors of cyclooxygenase-2 as nonulcerogenic anti-inflammatory agents. Curr Opin Chem Biol. 1998;2:482-490.

14 Busch U, Schmid J, Heinzel G, Schmaus H, Baierl J, Huber C, et al. Pharmacokinetics of meloxicam in animals and the relevance to humans. Drug Metab Dispos. 1998;26:576-584.

15 Davis A, Perkins MN. The effect of capsaicin and conventional analgesics in two models of monoarthritis in the rat. Agents Actions. 1993;38 Spec No:C10-12.

16 Warner TD, Giuliano F, Vojnovic I, Bukasa A, Mitchell JA, Vane JR. Nonsteroid drug selectivities for cyclo-oxygenase-1 rather than cyclo-oxygenase-2 are associated with human gastrointestinal toxicity: a full in vitro analysis. Proc Natl Acad Sci U S A. 1999;96:7563-7568. 